\title{
Fluctuation characteristic measurement for sound environment evaluation
}

\author{
Yasuhiko Tahara,* Kiminori Yamaguchi, ${ }^{* *}$ and Tohru Miyajima* \\ *Institute of Technology, SHIMIZU CORPORATION, \\ 3-4-17 Etchujima, Koto-ku, Tokyo, 135 Japan \\ **ONO SOKKI Co., Ltd., \\ 1-16-1 Hakusan, Midori-ku, Yokohama, 226 Japan
}

\begin{abstract}
In recent years, the attempt to evaluate the acoustic environment using the flucutuation characteristic (power spectral density of fluctuation) has been attracting attention. This paper introduces examples of fluctuation measurement aimed at environment evaluation and examines various problems related to fluctuation characteristic measurement of audio signals. Also, the function and composition of a special measurement system for measurement of the "fluctuation characteristic," the fluctuation analyzer, are described in the last part of the text. The authors have begun developing this system recently with the aim of supporting these studies.
\end{abstract}

Keywords: Comfortable environment, Fluctuation characteristic, Power spectral density, $1 / f$ behavior, Fluctuation analyzer

PACS number: 43.85 . $-\mathrm{e}$

\section{INTRODUCTION}

In Japan recently, stress in the work places has been increasing and the demand for a comfortable indoor environment has been growing more and more with the modernization of office-work conditions through introduction of data-processing equipment such as computers and word processors.

From the standpoint that comfort originates in the natural environment, the authors ${ }^{1)}$ have studied the fluctuation characteristics of the amplitude and frequency of various audio signals (such as natural sound, music, etc.) and the power spectral density of "a ridge line of mountain," "a breath of wind," etc., fixing their eyes on the fluctuating phenomena which are one of the important features of the natural environment. As a result, interesting characteristics were observed in the various objects of measurement. The fluctuating phenomena were found to represent an important aspect of environment and have possibilities for becoming a key factor in comfort evaluation.

R. F. Voss and J. Clarke, ${ }^{2)}$ who performed a pioneer study in this field, have proposed a methodology of fluctuation characteristic measurement and introduced several intersting findings indicating the importance of the fluctuation characteristic. However, the actual status of the fluctuation characteristic in the natural field is not thoroughly clarified yet, and there are many matters left to be solved regarding the measurement method for fluctuation characteristic and the evaluation method for fluctuation characteristic. Under these circumstances, and considering it necessary to observe the actual status of fluctuation in the natural environment further and in more detail to accumulate data, we have reached the idea of development of a special measurement system dedicated to the fluctuation characteristic, "the fluctuation analyzer," with the aim of supporting these studies.

In this paper, the measurement results of fluctuation characteristic which authors have performed so far are reported first, and then here various problems related to fluctuation characteristic measurement aimed at environment evaluation are examined. Also, in the last part of the text, the function and 
composition of the fluctuation analyzer, which the authors have started developing recently, are described.

\section{FLUCTUATION SPECTRAL DENSITIES FOR AUDIO SIGNAL}

Based on R. F. Voss's method, the measurement system shown in Fig. 1 is composed. Figure 1(a) is a measurement system for "audio power fluctuation," for which the power spectral density is obtained with an FFT analyzer, by determining the fluctuation components at and below $20 \mathrm{~Hz}$ by squaring audio signals. Figure 1(b) is a measurement system for "frequency fluctuation," for which the power spectral density is obtained by FFT operation, by inputting a time series of zero cross numbers of audio signals obtained as outputs of the frequency counter.

Typical examples of measured fluctuation characteristics are shown in Fig. $2 \sim$ Fig. 4 . In these figures, (a) shows the fluctuation of audio power and (b) the fluctuation of frequency.

Figure 2 analyzes the whole score of "Four Seasons" by Vivaldi (approx. $39 \mathrm{~min}$ ), Fig. 3 "BAD" by M. Jackson (approx. 4 min), and Fig. 4 a segment from Japanese "NHK News" (approx. 15 $\mathrm{min})$. Straght lines in these figures are regression lines of fluctuation characteristic waveform within a range of $0.05 \sim 1.0 \mathrm{~Hz}$, and coefficients of fluctuation $\left(n\right.$ of $\left.f^{n}\right)$ calculated from their inclinations are shown at the bottom left of each figure.

In case of Fig. 2, $n$ is near -1.0 for both audio power fluctuation and frequency fluctuation, indicating the typical $1 / f$ characteristic as has been stated so far. Since in the case of Fig. 3, $n$ is near a range of $-0.4 \sim-0.5$ in case of Fig. $4, n$ is near 0 , both are off the $1 / f$ characteristic.

In Fig. 5, coefficients $\left(n_{\mathrm{p}}\right)$ of audio power fluctuation and coefficients of frequency fluctuation $\left(n_{\mathrm{f}}\right)$ by 20 typical audio signals are plotted on a two dimensional plain by omitting their minus signs.

Table 1 shows the specific contents of these 20

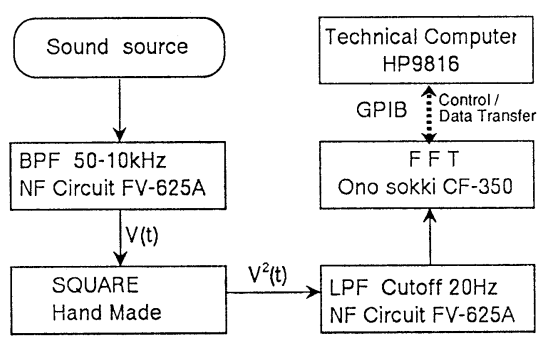

(a) Audio power fluctuation

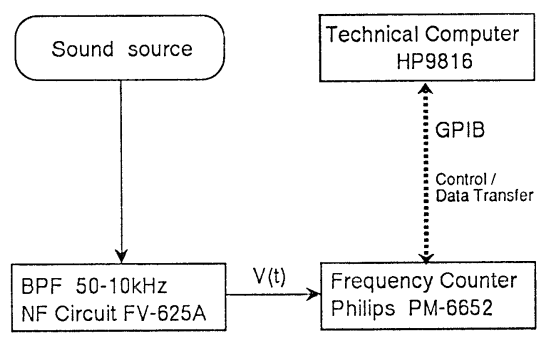

(b) Frequency fluctuation

Fig. 1 Block diagram of a fluctuation measurement system for audio signals.
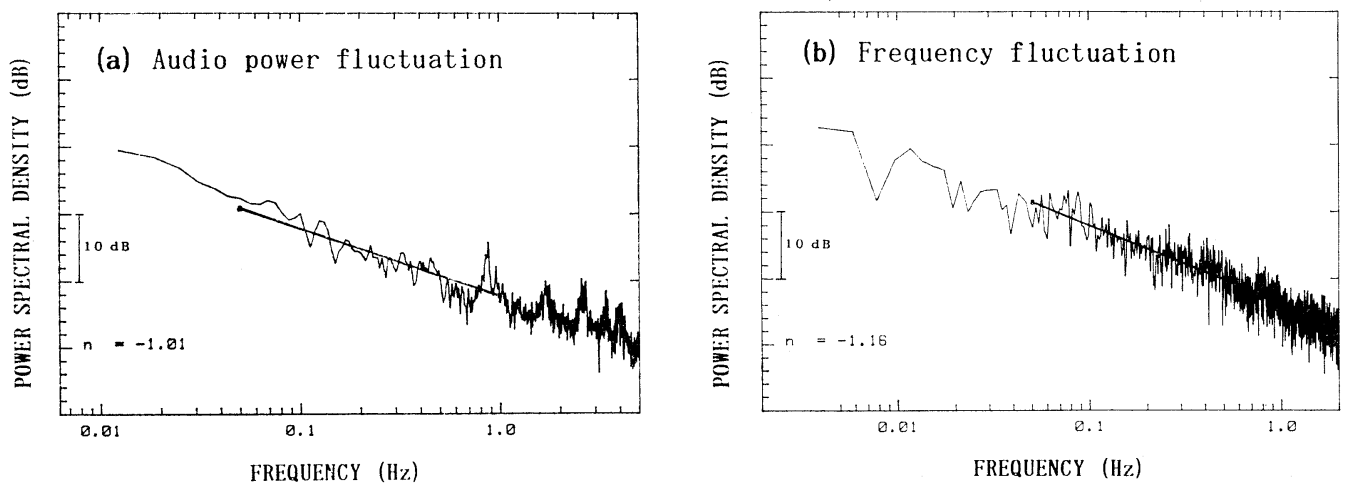

Fig. 2 Fluctuation characteristics for Vivaldi's "FOUR SEASONS" (39 min). 


\section{Y. TAHARA et al.: FLUCTUATION CHARACTERISTIC MEASUREMENT}
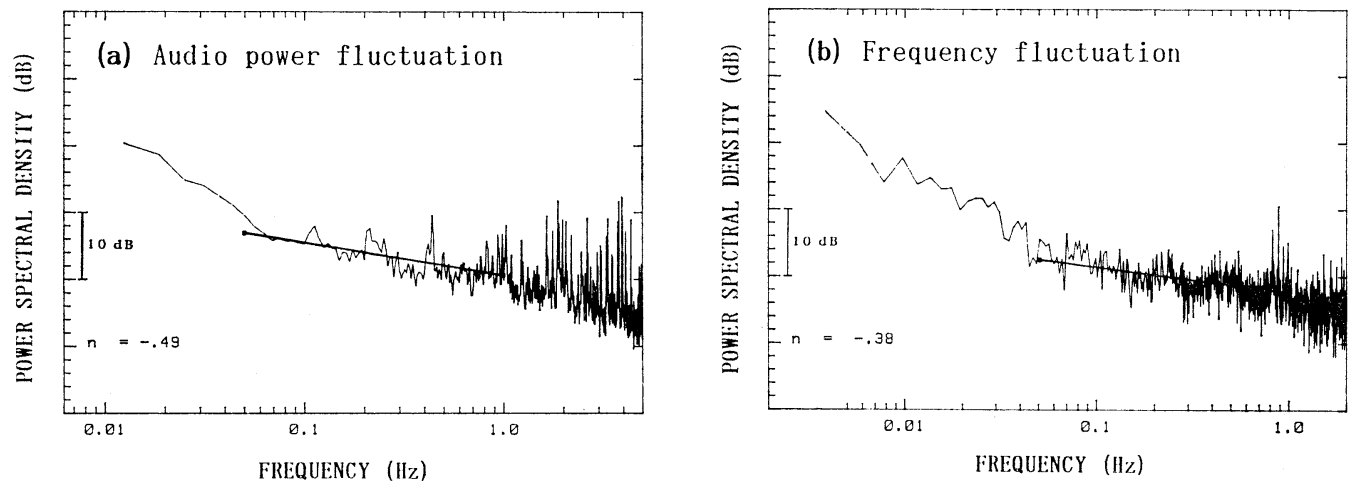

Fig. 3 Fluctuation characteristics for Michael Jackson's "BAD" (4 min).
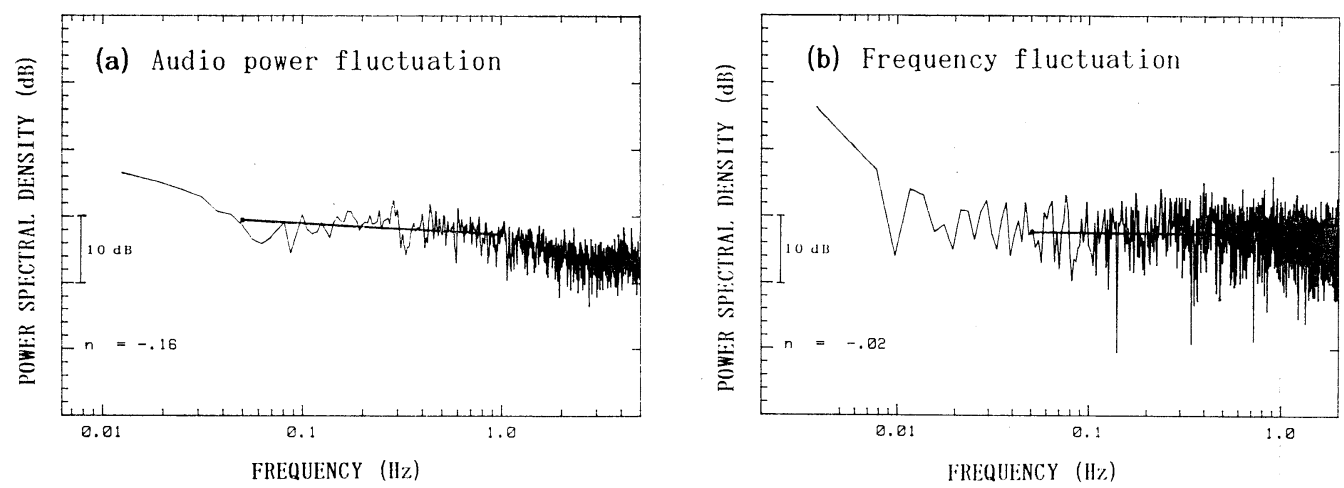

Fig. 4 Fluctuation characteristics for "NHK Radio News" (15 min).

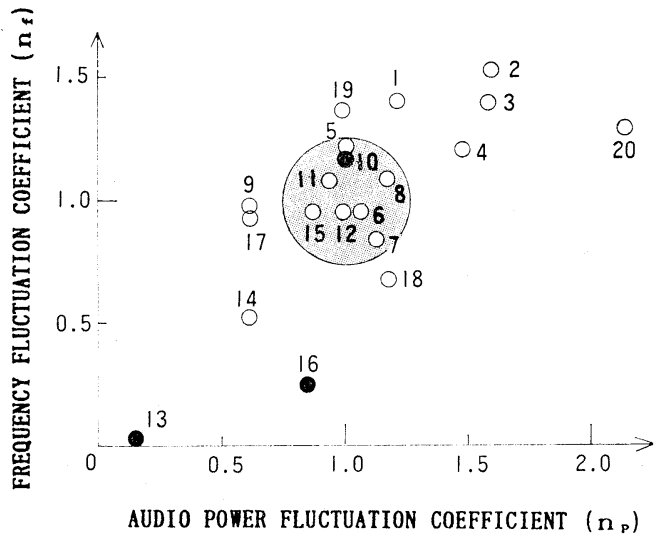

Fig. 5 Two dimensional representation of fluctuation coefficients (black dots correspond to the audio signals in Fig. 2 Fig. 4). typical audio signals shown in Fig. 5. In Fig. 5, a circle with a 0.25 radius is centered at $n_{\mathrm{p}}=n_{\mathrm{f}}=1.0$. As a guideline, audio signals within this circle can be considered to have a typical $1 / f$ characteristic. According to the figure, the Japanese news (No. 13) and the sea wave (No. 20) are direct opposite types in regard to fluctuation characteristic, and much classic music present a typical $1 / f$ characteristic. There may be various opinions about how to read the fluctuation characteristic data of Fig. 5. Combining the results of measurements performed by the authors, fluctuation characteristic is assumed to most probably contain information of extreme importance related to the bioaffinity of audio signals, and continued systematic data accumulation will be useful in the future as well. 


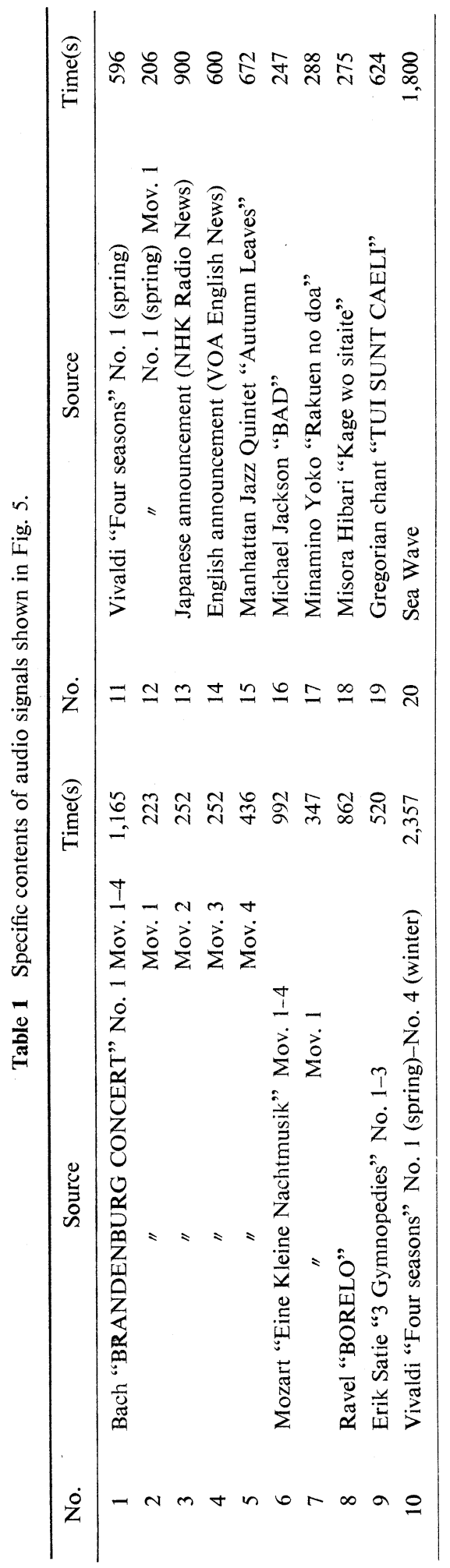

\section{FLUCTUATION SPECTRAL DENSITIES FOR NATURAL PHENOMENA}

Here, results of spectral density analyses related to "ridgelines of mountains" and "a breath of wind" are shown as an example of the fluctuation characteristic of general natural phenomena.

Figure 6 shows the ridgeline of the whole somma of a well-known caldera volcano (Mt. Aso) in Japan. When the power spectral density is determined from the ridgeline waveform as a time series signal, a gentle waveform descending to the right is obtained as shown in Fig. 7. Figure 7 shows the typical $1 / f^{2}$ characteristic $(n=-2)$ of audio signals which induce relaxation and drowsiness. This is quite an interesting result when considered together with the grand and gentle shape of this mountain.

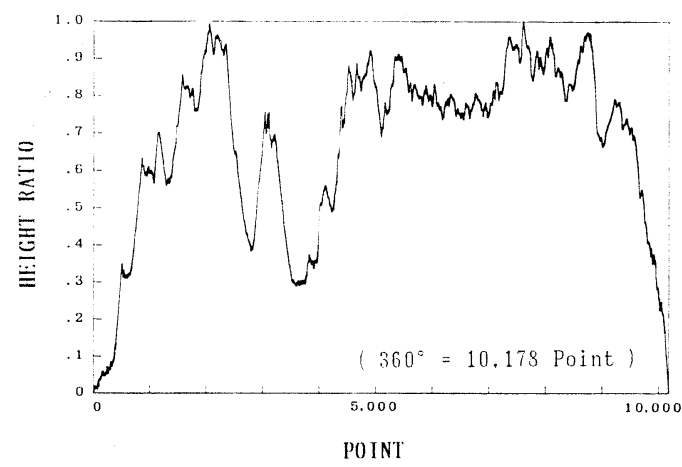

Fig. 6 The somma ridgeline of "Mt. ASO" in Kumamoto Japan.

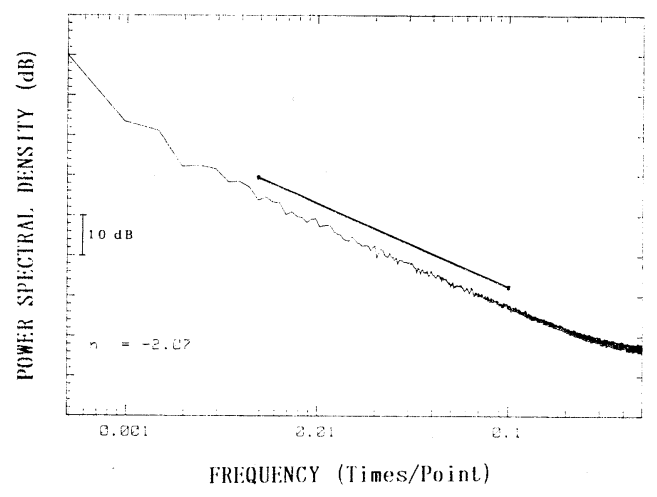

Fig. 7 Power spectral density for the somma ridgeline of "Mt. ASO." 


\section{Y. TAHARA et al.: FLUCTUATION CHARACTERISTIC MEASUREMENT}

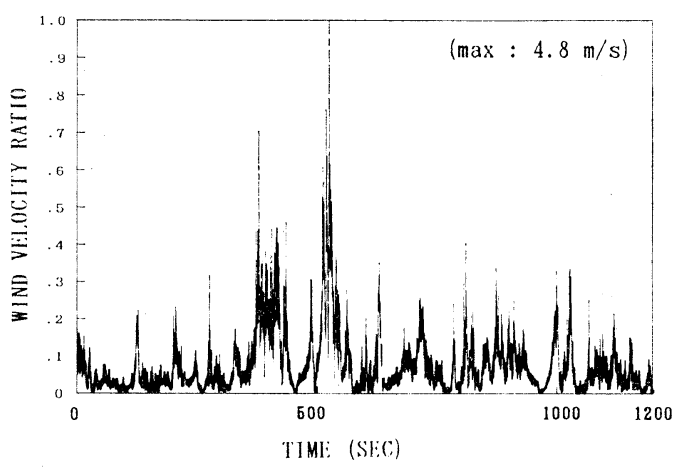

Fig. 8 Relative wind velocity of breeze observed in the woods of Karuizawa Japan.

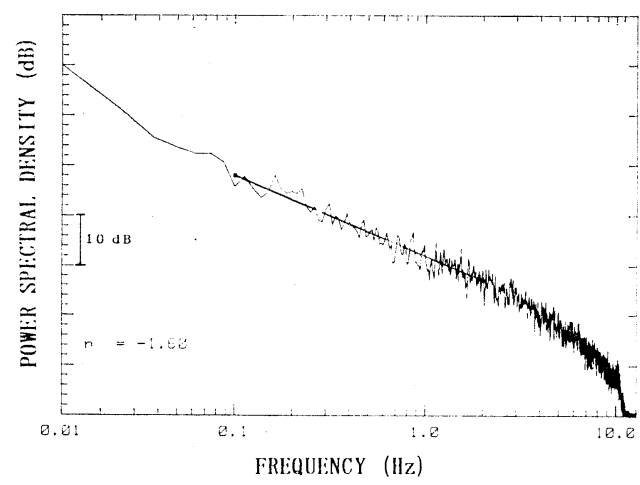

Fig. 9 Power spectral density for wind velocity of breeze in Karuizawa.

On the other hand, Fig. 8 shows a relative wind velocity, displayed as a time series signal for $20 \mathrm{~min}$, of breeze observed in the woods of Karuizawa. Figure 9 is the power spectral density, presenting a gentle and simple decreasing waveform with $n=$ -1.6. This value of $n=-1.6$ corresponds to $f^{-5 / 3}$ (a spectrum of soft breeze) which is known well in the field of fluid dynamics, and this can be said to be a typical example showing that a comfortable natural fluctuation is not always equal to $1 / f$.

Above, two sample measurements of fluctuation characteristic related to natural phenomena other than sound were introduced. Each of these cases shows a simple and clear fluctuation characteristic (power spectral density) regardless of its complication of original waveform, and it can be considered an indication that fluctuation characteristic rep- resents important characteristics of the natural fields.

\section{DISCUSSION ON FLUCTUATION MEASUREMENT FOR ENVIRONMENT EVALUATION}

Beginning with a study done by R. F. Voss and J. Clarke, ${ }^{2)}$ many researchers have shown interest in fluctuating characteristic, and useful data has been accumulated so far. However, the fluctuation characteristic which is the power spectral density of the fluctuation, expresses only one aspect of fluctuating phenomena, and so it is necessary to clarify the "center of fluctuation (average value)" and the "amplitude of fluctuation" in addition to the fluctuation characteristic in order to reproduce the fluctuating phenomena in another space. Moreover, in the fluctuation, in addition to the amplitude (audio power) fluctuation and the frequency fluctuation presented by Voss, there is an interval fluctuation of intermitted physical phenomenon (i.e., "a period fluctuation") which is represented by the fluctuations of calls of various birds and of the heartbeat cycle. T. Musha, ${ }^{3)}$ a noted researcher in this field in Japan, has found a quite interesting phenomenon on biological period fluctuation in which the fluctuation of time interval for heartbeat, spontaneous spike discharge of a newron and a free clapping rhythm show $1 / f$ characteristic in many cases. However, the data accumulation on the period fluctuations has been delayed compared with the former two.

The reason that the fluctuation characteristic is expected to have significance as an index of environment evaluation is because the safety performance of the natural field, such as information related to peace and safety, natural calamity, attack of an enemy, etc., is thought to be included in the fluctuation characteristic and it is thought that the memories are written deep in human's brains. Consequently, in regard to the fluctuation characteristic measurement for environment evaluation, it is considered importance to specify the meaning of any objective physical quantity viewed from the living bodies' standpoint. From this viewpoint, the fluctuation measurement of audio signals contains various problems such as (1) necessity or lack thereof of auditory sense adjustment of input signal, (2) relationship of audio power (square sound pressure) and loudness, (3) appropriateness of the frequency fluctuation measurement using zero cross rate, and 
(4) method of determining fluctuation coefficients $n$ (range of a fluctuation frequency to be focused on), etc.

Because of the present situation described above, it is necessary to try to solve various problems in the systematic accumulation of fluctuation characteristic data and in the fluctuation measurement technics. The measurement means of the fluctuation characteristic has a very important meaning in promoting these. Thus, the authors have reached the idea of developing a "fluctuation analyzer" which is applicable to each of these three measurement items, namely "amplitude fluctuation," "frequency fluctuation," and "period fluctuation" and simultaneously operates and display three fluctuation parameters of "fluctuation characteristic," "fluctuation center" and "size of fluctuation." Also, this fluctuation analyzer is designed with consideration for measurement of other various phenomena beside sound, based on experience in measuring the fluctuation characteristic associated with mountain ridges and a breath of wind.

\section{HARDWARE IMPLEMENTATION OF FLUCTUATION ANALYZER}

Here, the function and composition of the "fluctuation analyzer," which the authors have begun developing recently, are described.

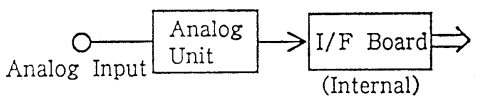

The construction of a personal computer-based digital fluctuation analyzer is shown in Fig. 10. It consists of two major parts: the host computer unit and the analog unit which intercommunicates through the $\mathrm{I} / \mathrm{F}$ board.

Figure 11 shows the block diagram of this fluctuation analyzer. The analog unit contains two analog processing systems: one is used for obtaining the fluctuation of audio power or the fluctuation of event-time interval (period fluctuation), and the other is used for obtaining the fluctuation of frequency. The analog input signal is converted by the 16-bit $\mathrm{A} / \mathrm{D}$ converter or the counter into the digital form and sent to the PC through the $\mathrm{I} / \mathrm{F}$ board. The coefficients of fluctuation are calculated from the inclinations of the power spectral density of the signal, which is computed by FFT method by applying the method of least squares, and other statistical values such as mean value and standard deviation of fluctuations are also computed.

The processed input signal and the computed results can be monitored by the display unit, and they are also recorded by the printer connected to the PC. The processing descriptions for three types of fluctuation, fluctuation of audio power, fluctuation of frequency and fluctuation of eventtime interval (period fluctuation), are shown in Figs. 12, 13 and 14.

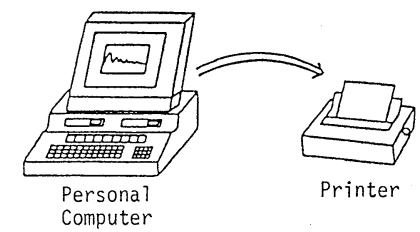

Fig. 10 Construction of a PC-based fluctuation analyzer.

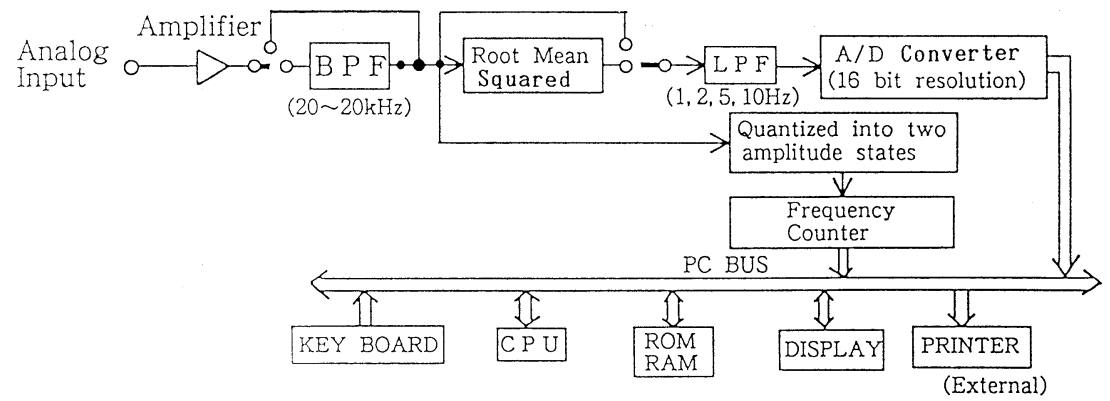

Fig. 11 Block diagram of the fluctuation analyzer. 


\section{Y. TAHARA et al.: FLUCTUATION CHARACTERISTIC MEASUREMENT}

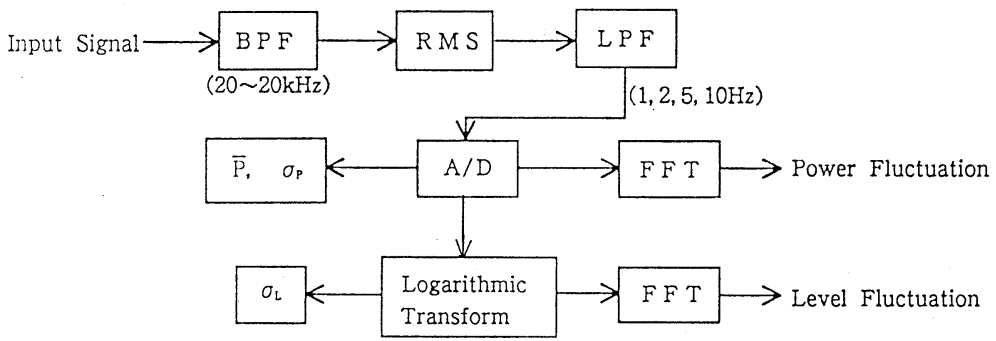

Fig. 12 Processing description for the fluctuation of audio power.

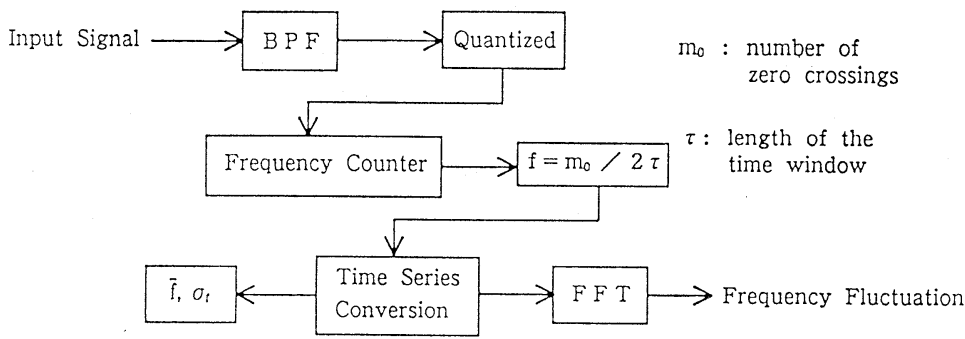

Fig. 13 Processing description for the fluctuation of frequency.

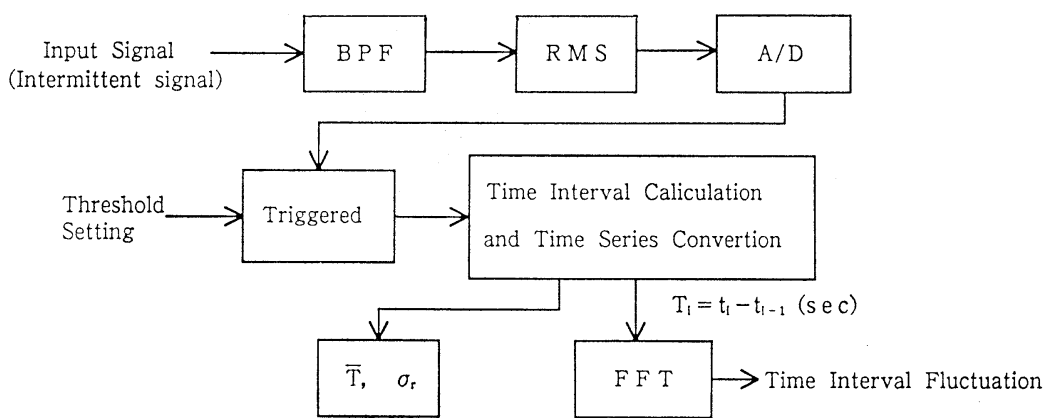

Fig. 14 Processing description for the fluctuation of event-time interval.

\section{CONCLUSION}

Examples of fluctuation characteristic measurement for environment evaluation were introduced and various problems related to the fluctuation characteristic measurement were examined. Also, for the last, the function and composition of a special measurement system dedicated to the fluctuation characteristic, the fluctuation analyzer, which the authors have recently started developing to support these studies, were described.

The attempt to evaluate the environment through research using the fluctuation characteristic has just started recently, and it contains many subjects to be solved. For the future, the role of measurement means is very important for developing the study of this area.

The fluctuation analyzer which is now under development is a computer-based digital system, and it will provide high adaptability, high accuracy, and highly efficient means for analyzing the fluctuating behavior of sound. Through measurements of acoustic environment using this fluctuation analyzer, more findings than ever can be expected. Moreover, it can be expected that this fluctuation analyzer will be applied not only in the acoustic 
field but also in all field of environmental engineering including temperature and heat, scenery, wind, flavor, etc.

\section{ACKNOWLEDGMENTS}

The authors would like to thank $\mathrm{H}$. Imaizumi, $\mathrm{T}$. Musha, K. Sawatari of the Acoustics Dept., ONO SOKKI Co., Ltd., and Y. Sato of OEG Co., Ltd., for their support in the development of the fluctuation analyzer.

\section{REFERENCES}

1) T. Miyajima and Y. Tahara, "Fundamental study on sound environment control using sound adding technique-Measuring method and measured results for fluctuation characteristic of audio signal," Proc. Autumn Meet. Acoust. Soc. Jpn., 697-698 (1988) (in Japanese).

2) R. F. Voss and J. Clarke, " ' $1 / f$ noise' in music: Music from $1 / f$ noise," J. Acoust. Soc. Am. 63, 258 263 (1978).

3) T. Musha, "Music and heartbeat rate," 1 st Int. Conf. Music Perception and Cognition, Kyoto, Japan, 281-286 (Oct. 1989).

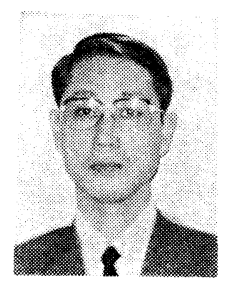

Yasuhiko Tahara received M. Eng. and Dr. Eng. degrees in 1969 and 1987, respectively, from Tohoku University, Japan. Since April 1969, he has worked at Institute Technology, SHIMIZU CORPORATION, where he is a Chief Research Engineer. He has been engaged in research on noise control, room acoustics and indoor environment evaluation, and in acoustical design of recording studios and concert halls.

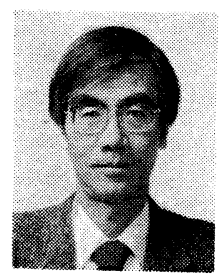

Kiminori Yamaguchi was born in Gunma, Japan on December 14, 1940. He received B.E. and Dr. Eng. from Tohoku University, Sendai, Japan, in 1964 and 1978 respectively. In 1984, he joined ONO SOKKI Co., Ltd., and now he is a director of ONO SOKKI Co., Ltd. $\mathrm{He}$ has been engaged in developing measuring instruments for sound and vibration. Dr. Yamaguchi is a member of ASJ, ASA, AIJ and INCE Japan.

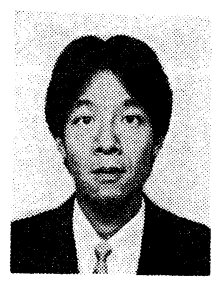

Tohru Miyajima was born in Fukuoka, Japan, on March 7, 1963. He received B.E. degree from Kyushu Institute of Design, Fukuoka, Japan in 1986. He joined Institute of Technology, SHIMIZU CORPORATION, in 1986. Since then he has been working on auralization of sound field. Mr. Miyajima is a member of Acoustical Society of Japan. 